\title{
Single, rapid coastal settlement of Asia revealed by analysis of complete mitochondrial genomes
}

\begin{abstract}
A recent dispersal of modern humans out of Africa is now widely accepted, but the routes taken across Eurasia are still disputed. We show that mitochondrial DNA variation in isolated ñelictò populations in southeast Asia supports the view that there was only a single dispersal from Africa, most likely via a southern coastal route, through India and onward into southeast Asia and Australasia. There was an early offshoot, leading ultimately to the settlement of the Near East and Europe, but the main dispersal from India to Australia $\sim 65,000$ years ago was rapid, most likely taking only a few thousand years.
\end{abstract}

Keyword: Coastal settlement; Mitochondria; Mitochondrial DNA; Mitochondrial genome 